\title{
ESTABLISHING A SENSORY TESTING \\ LABORATORY, PANEL AND PROCEDURE FOR \\ BISCUIT INDUSTRY
}

\author{
BY \\ D.H.VINOY JAYASHANTHA
}

A thesis to the University of Sri Jayawardenepura for the award of the degree

$$
\text { Of }
$$

Master of Science

In

Food Science and Technology

Year 2006 


\section{Declaration}

The work described in this thesis was carried out by me under the supervision of Prof. A. Bamunuarachchi and Dr. K.K.D.N.S. Ranaweera and a report on this has not been submitted in whole or in part to any University or any other institution for another Degree

\section{/ Diploma.}

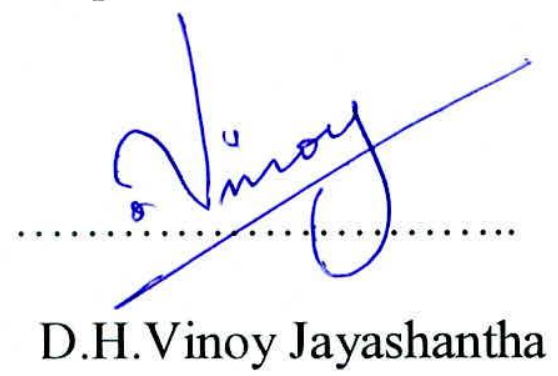




\section{Declaration}

"We certify that the above statement made by the candidate is true and that this thesis is suitable for submission to the University for the purpose of evaluation"

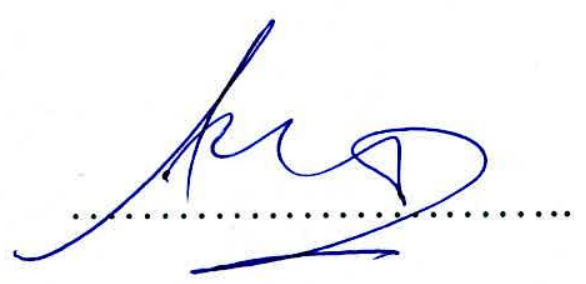

Prof. A.Bamunuarachchi

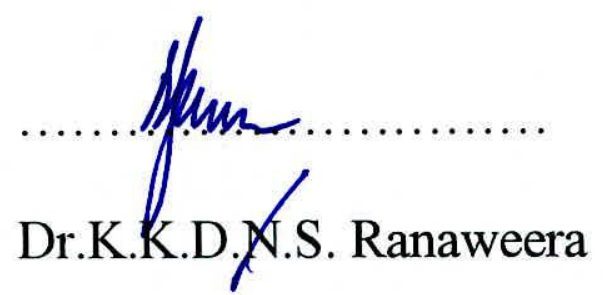




\section{ACKNOWLEDGEMENT}

I wish to express my sincere appreciation for the advice, guidance and suggestions so willingly given by my supervisor Prof. A. Bamunuarachchi and Dr. K.K.D.N.S. Ranaweera of the Department of Food Science, University of Sri Jayewardenepura ,for all their guidance, assistance and encouragement given to me in making this research project success.

I express my heartiest gratitude to the Management of Maliban Biscuit Manufactories limited for granting me all the necessary arrangements. I also express my special thanks to Mrs. Varna Fernando for the facilitation and guidance given for me in making this research project success.

I express my sincere thanks to all the staff members and all other members of the Maliban Biscuit Manufactories limited for the active participation and the support extended to me during this project. 


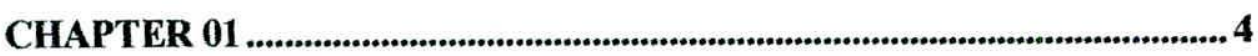

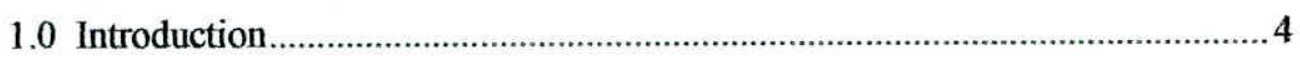

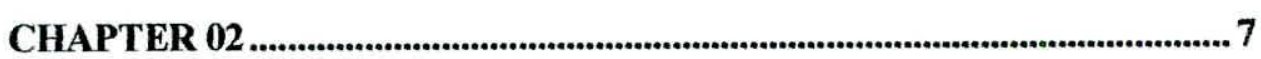

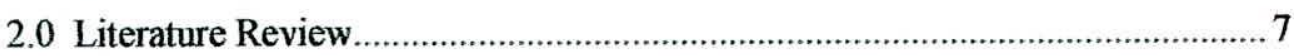

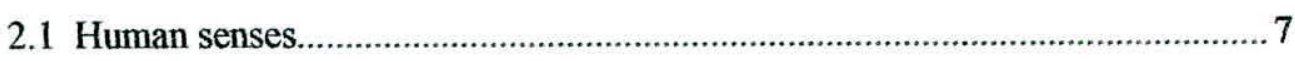

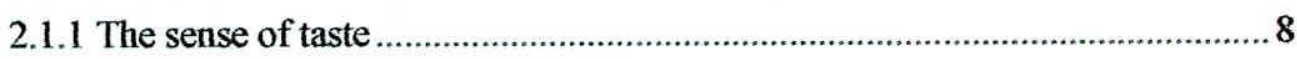

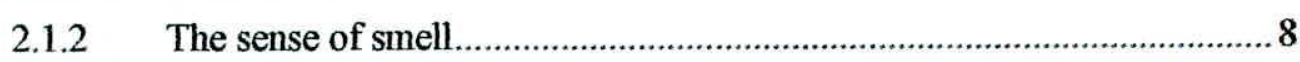

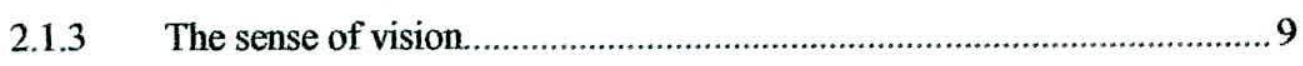

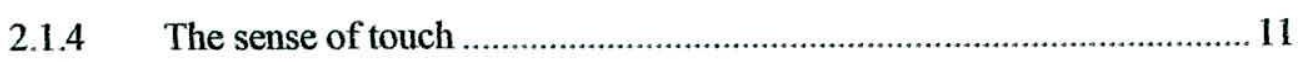

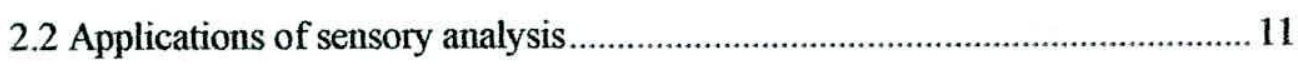

2.3 Classification of biscuits ............................................................................ 14

2.3.1 Classification based on enrichment of the recipe ................................ 14

2.3.2 Classification based on method of dough piece formation ..................... 16

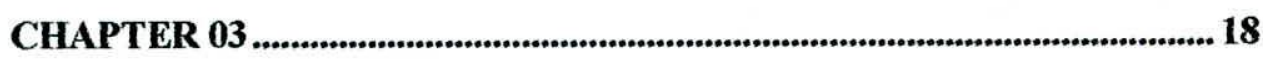

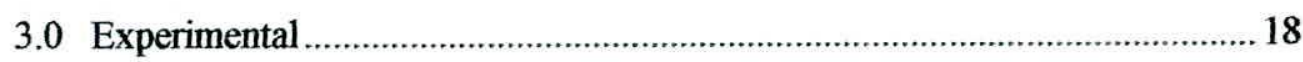

3.1 Establishment of Sensory Testing Laboratory .............................................. 18

3.1.1 Designing of sensory testing laboratory ................................................ 18

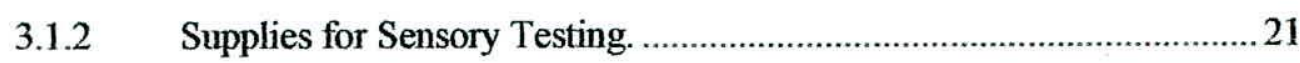

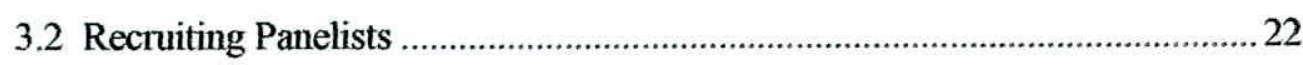

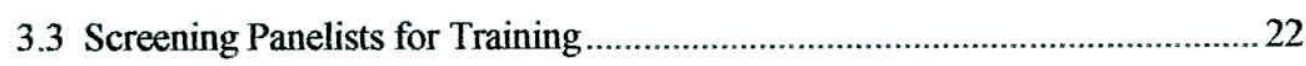

3.3.1 Initial Screening for General Sensory Acuity ...........................................22

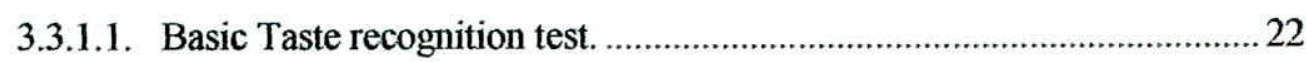

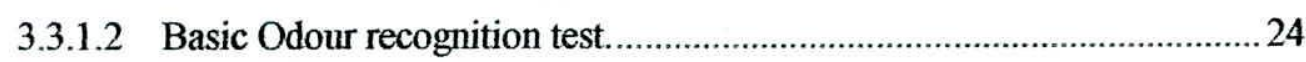

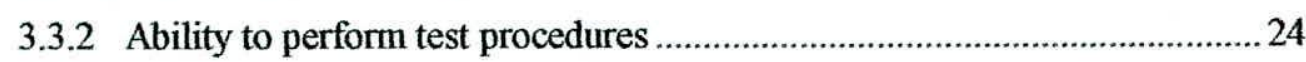

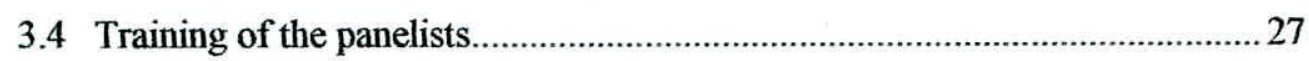

3.4.1 Identify a Difference Using a Triangle Test .........................................2

3.4.2 Ranking for intensity of a sensory characteristic...............................2 29

3.4.3 Scoring for perceived intensity of specific characteristic ............................ 30

3.4.4 Descriptive test for determine the intensities for given sensory profile ....... 31

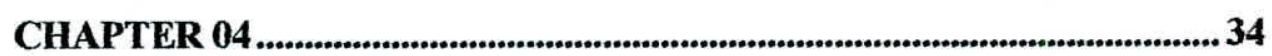

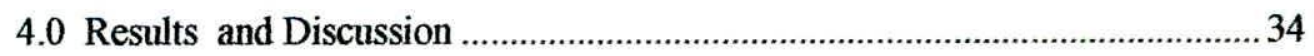


4.1 Establishment of Sensory Testing Laboratory ............................................... 34

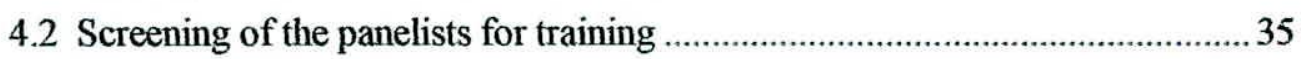

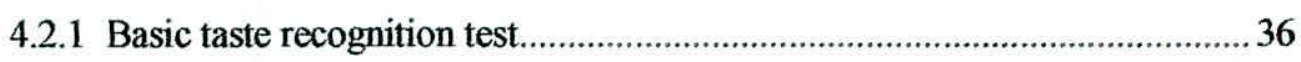

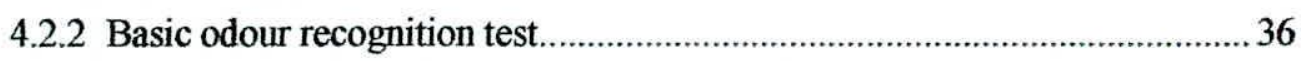

4.2.3. Criteria for selection for Sensory Panel .................................................... 37

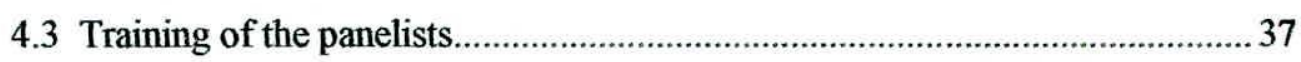

4.3.1 Identify a Difference Using a Triangle Test .......................................... 38

4.3.2. Ranking for intensity of a sensory characteristic.................................... 41

4.3.3.1. Scoring for perceived intensity of specific characteristic .........................44

4.3.3.2. Scoring for perceived intensity of specific characteristic ......................... 49

4.3.3.3 Scoring for perceived intensity of specific characteristic ...........................55

4.3.4 Descriptive test for determine the intensities for given sensory profile ......60 60

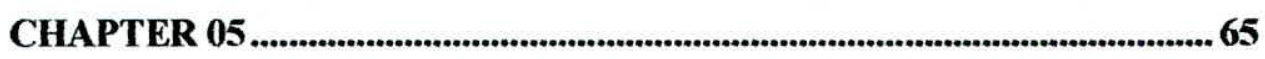

5.0 Conclusion.................................................... 65

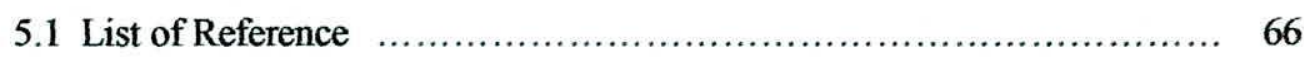




\section{Table of Figures}

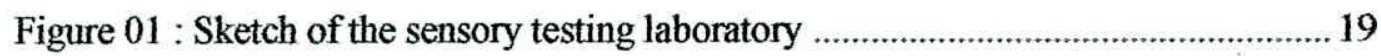

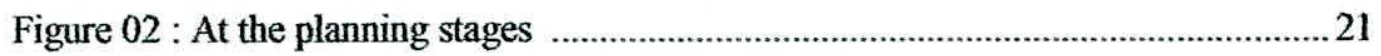

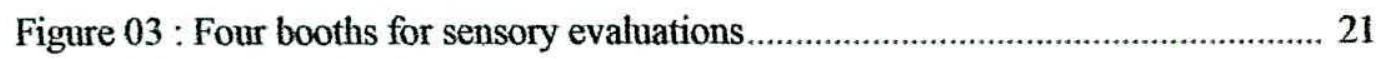

Figure 04 : Food preparation area with Sink \& water supply. ...................................... 22

Figure 06 : Basic taste solutions \& water for taste recognition test ...............................2 27

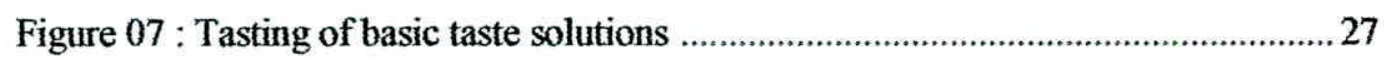

Figure 08 : Vials used for the common odour recognition test ................................27

Figure 09 : Panelists are being properly instructed about the objective and

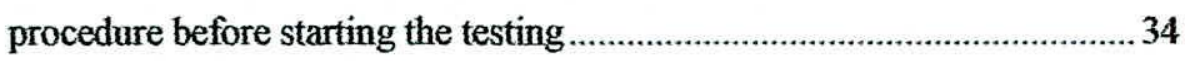

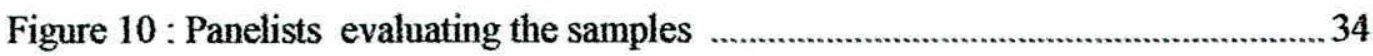

Figure 11 : Performance of panelists are closely monitored during the session............. 34

Figure 12 : Panelist performing Traingular Test ....................................................... 34 


\begin{tabular}{|c|}
\hline Apendices \\
\hline Appendix 01 \\
\hline Ballot paper 01 \\
\hline Ballot paper 02 ........... \\
\hline Ballot paper $03 . . .$. \\
\hline 70 \\
\hline Ballot paper $05 . . . . . . .$. \\
\hline Ballot paper 06 \\
\hline 73 \\
\hline 74 \\
\hline 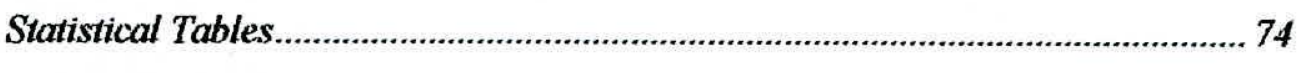 \\
\hline Appendix 03 \\
\hline Reference Standal \\
\hline
\end{tabular}




\title{
Establishment of Sensory Evaluation \\ Laboratory,Panel and Procedures
}

For

the Biscuit Industry

by

\section{D.H.V.JAYASHANTHA}

\begin{abstract}
This study was done to establish sensory testing laboratory, panel and procedures for the biscuit industry. Informal sensory evaluations may provide good opportunities for biased opinions, observations and comments .Therefore it was needed to have properly structured frame work of sensory analysis for getting reliable, accurate and repeatable results which may be important in critical business decisions that are highly dependent upon assessment of product quality. Sensory testing laboratory was established with basic requirements and 50 members from the entire company were recruited and 20 members out of 50 were selected after several screening tests. Four procedures were developed to evaluate biscuits with different objectives and trained the panelists for developed procedures. The sensory analysis results give boost to the research and development studies with quantification of sensory characteristics. Sensory evaluations also help in assurance of product quality and product acceptance.
\end{abstract}




\section{CHAPTER 01}

\subsection{Introduction}

Sensory analysis is the identification, scientific measurement, analysis and interpretation of the properties (attributes) of a product as they perceived through the five senses of sight, smell, taste touch and hearing.

Sensory characteristics are more important than most of the other factors. Foods are complex mixtures of organic and inorganic compounds. During the consumption various physico-chemical characteristics of the foods stimulate all of our senses to some extent. These stimulations are those such as saltiness, sweetness, redness, toughness, acidity,bitterness,astringency,cold,hot,crisipiness, hardness,adhesiveness, elasticity,iciness,viscocity,size,shape,opacity,gloss,lightness, bluness,greeness, redness, earthiness, etc.

Today the world over the food industry tends to rely on sensory evaluation for the confirmation of their products for customer satisfaction. There are some barriers associated with obtaining definite sensory information from informal tasting sessions. Commonly they as follows

1 Setting the wrong objective

2 Asking from wrong participant

3 Asking wrong questions

4 Biased judgment

5 Lack of scientific control

6 Wrong environment

By doing formal sensory analysis we can eliminate the above pitfalls. The formal sensory evaluation should include setting of right objectives, asking from right people 
(i.e. trained sensory panel), asking right questions and scientific control under control environment.

In this research project three objectives were set to overcome the above barriers associate with the sensory analysis of biscuits.

1) Establishment of sensory testing laboratory

2) Establishment of sensory testing Panel

3) Establishment of sensory testing Procedures

\section{Biscuits}

Biscuits are small baked products made principally from flour, sugar and fat. They typically have a moisture content of less than $4 \%$ and when packaged in moisture proof containers have a long shelf life, perhaps almost one year. Also it contains less water activity value which retards the growth of micro organisms. ( aw $<0.4$ )

The acceptance by consumers is determined by the appearance and eating qualities. For example, consumers do not like broken biscuits over or under baked biscuits, biscuits with foreign matter, soggy biscuits etc.Therefore sensory characteristics are very important characteristics in this industry. It is make essential to have well established sensory system in order to deligt the customer in the fast growing market with enormous competency in Sri Lankan \& other countries.

Types of biscuits;

1. Cracker type biscuits (eg. Cream Cracker)

2. Marie type biscuits (eg. Gold Marie)

3. Cookies (eg. Butter Cookies)

4. Savory type biscuits (eg. Krisco, Cheese Bits etc.) 


\section{Sandwiched type biscuits (eg. Chocolate Cream )}

6. Biscuits coated with chocolates (eg. Chocolate fingers)

Biscuits are classified due to the origin as hard dough or soft dough. The hard dough groups are savory, unsweetened, or semi-sweet and include all types of Crackers, puff dough biscuits and the semi-sweet varieties such as Marie type.

The soft dough group includes all the sweet biscuits, whether they are plain biscuits, shells are flow type such as ginger nuts. Soft dough biscuits all have many factors in common, but hard dough biscuits fall naturally into three categories. Fermented doughs, puff doughs and the semi sweet doughs. 


\section{CHAPTER 02}

\section{Literature Review}

Sensory analysis is the identification, scientific measurement, analysis and interpretation of the properties (attributes) of a product as they are perceived through the five senses of sight, smell, taste, touch and hearing.

\subsection{Human senses}

1. Gustatory - sense of taste

2. Olfactory sense - sense of smell

3. Visual sense - Vision

4. Audition - Sense of Hearing

5. Somesthetic sense-Sense of Touch

Perception plays a major role in the science of sensory analysis. Both psychology and physiology play an important part in the process of sensory analysis. When we consume food stuffs, it may use some or all five of their sense - sight, smell, taste, hearing and touch.

\subsubsection{The sense of taste}

There are several sections of the tongue which specialized in sensing different tastes. The sweet is most easily sensed at the tip of the tongue, the saline at the tip and edge, the sour at the edge, and the bitter at the back. The papillae for bitter are especially deep, and so this sensation takes longer to perceive but tends to get longer time to going away. 
The sensation of taste is a result of the effect of water soluble molecules interacting with receptors on the tongue and in the oral cavity. These receptors contain taste buds that are renewed every six to eight days. Taste substances are received onto the membranes of those cells containing taste buds, which then transmit an impulse to the brain.

\subsubsection{The sense of smell}

The nose of human can detect lot of odors. Human nasal cavity is divide into two spaces, left and right by a partition called the nasal septum .Each of these spaces folds to form ridges, called conchae, which project in to the interior from the outside walls of the nose ..The entire nasal cavity is lined with a mucous membrane. The smell receptor cells ,olfactory sensory cells, are restricted to olfactory region. The part of the nasal mucosa that lacks olfactory cells is the respiratory region, which has a ciliated epithelium producing the mucus.

The olfactory epithelium is responsible for the sense of smell. there are two predominated cell types here, the olfactory sensory cells and the supporting cells .Basal cells are also present as in the taste buds and serve the same function to regenerate the sensory cells. The olfactory sensory cells bear cilia towards the olfactory epithelium and are covered by a layer of mucus in order for an odor to reach the sense cells, the molecules of an odor substance must diffuse thought the mucus layer. Unlike the taste receptor cells, the olfactory cells are primary sensory cells in that they send out axons from the basal pole. There are so many (thousand)of odor substances that it is unlikely that there exists a particular sensory molecule for each odor substance. The known recognition of individual odor substances and the response of receptor cells is not known. However, it is known that olfactory sensory cells exhibit characteristic response 
spectra in that each single receptor cell is excited to different degrees by many substances. The relative sensitivity to various substances, at given concentrations, varies from one cell to another

\subsubsection{The sense of vision}

The vision is the first sensory character which deals severely to the acceptance or rejection of the food. Approximately $70 \%$ of all sensory receptors in the human body are located in the eyes. Light entering the eye passes through the lens and is focused on the retina. The retina is the sensory tunic of the eye and consists of two layers, the pigmented layer and the nervous layer. It is within the nervous layer that photoreceptors process light stimuli to form our vision. These photoreceptors, rods and cones, sense and encode the patterns made by light in our surroundings. Rods are responsible for dim light and peripheral vision and are more sensitive to light than cons. However they do not provide either sharp imagers or color vision. As light excites these photoreceptor cells, electrical signals are sent though sensory neurons of the bipolar cells and ganglion cell axons to the bundle of never fibers called the sensory neurons of the bipolar cells and ganglion cell axons to the bundle of nerve fibers called the optic nerve. This nerve from each eyeball is responsible for sending the signals to the brain (Marieb, E.N)

1) As visible light passes thought a prism, the waves are bent to produce a band of colors called the visible spectrum. This spectrum progress from red to violet. Objects thus have color because they absorb some wavelengths and reflect others. White objects reflect all wavelength of light and black objects absorb all wavelengths. There are some concerns in colour perception in sensory evaluation (Nancy L. DeVore : Sensory Physiology). They are as follows; 